\title{
Fundamentals of Cluster-Centric Content Placement in Device-to-Device Networks
}

\author{
Mehrnaz Afshang, Harpreet S. Dhillon, and Peter Han Joo Chong
}

\begin{abstract}
We develop a comprehensive analytical framework with foundations in stochastic geometry to characterize the performance of cluster-centric content placement in a deviceto-device (D2D) network. Different from device-centric content placement, cluster-centric placement focuses on placing content in each cluster such that the collective performance of all the devices in each cluster is optimized. Modeling the locations of the devices as a Poisson cluster process, we first show that the coverageoptimal performance can be achieved by placing content of interest for each cluster at the device closest to its cluster center. Since this restricts the frequency reuse within a cluster to one, and may not also be feasible due to storage/energy limitations, we also characterize the network performance in terms of coverage probability and area spectral efficiency (ASE) for a general class of strategies in which the content of interest for the typical device is available at the $k^{t h}$ closest device to the cluster center. Our analysis concretely demonstrates significant improvement in network performance when the content of interest for the typical device is biased to lie closer to the cluster center compared to the case when it is placed at a randomly chosen device.
\end{abstract}

Index Terms-D2D caching, optimal content placement, clustered D2D network, Thomas cluster process, stochastic geometry.

\section{INTRODUCTION}

Driven by the increasing mobile data traffic, cellular networks are undergoing unprecedented paradigm shift in the way data is delivered to the mobile users. A key component of this shift is device-to-device (D2D) communication in which proximate devices can deliver content on demand to their nearby users, thus offloading traffic from often congested cellular networks [1]. This is facilitated by the spatiotemporal correlation in the content demand (e.g., see [2]), which means that when the content is downloaded by one user from the network it can be locally cached and then delivered on demand to proximate users over direct links when those users need it [3]-[7]. This is termed as asynchronous content reuse in a cache-enabled D2D network. The performance of this network depends both on the locations of the D2D nodes and how the content is placed on those nodes. For instance, if the content is placed somewhat arbitrarily in the network, the distance between a D2D receiver and its potential transmitter (device having the content that is of interest to the receiver) may be large, which may lead to a poor D2D link performance. On the other hand, if the content is placed closer to a D2D receiver, the performance would be significantly better. In this

M. Afshang is with Wireless@VT, Department of ECE, Virgina Tech, Blacksburg, VA, USA and is with School of EEE, Nanyang Technological University, Singapore. Email: mehrnaz@vt.edu. H. S. Dhillon is with Wireless@VT, Department of ECE, Virgina Tech, Blacksburg, VA, USA Email: hdhillon@vt.edu. P. H. J. Chong is with School of EEE, Nanyang Technological University, Singapore. Email: Ehjchong@ntu.edu.sg. paper, we focus our attention on the cluster-centric content placement strategies with the goal of optimizing the collective performance of all the devices in the network

Related work. Modeling and analysis of D2D networks has taken two main directions. In the first line of work, the focus is more on the grid-based clustering models where the space is typically tessellated in square cells with devices in each cell forming distinct clusters [5], [6], [8]. To maintain tractability, several simplifying assumptions are made, e.g., simplified physical layer is assumed in which the communication between two nodes is possible only if the D2D link distance is smaller than some fixed collaboration distance. In the second line of work, the focus is on the accurate modeling of the physical layer metrics, such as the received signal to interference ratio (SIR) by using tools from stochastic geometry [7], [9]-[13]. To lend tractability, the locations of D2D transmitters (D2D-Txs) are typically modeled by a Poisson Point Process (PPP). On the other hand, the locations of D2D receivers (D2D-Rxs) are modeled by two approaches: i) D2D-Rxs lie at a fixed distance from their intended D2D-Txs [9]-[11], or ii) D2D-Rxs are uniformly distributed in the circles around their intended D2D-Txs [12], [13]. Unfortunately, neither of these models capture the notion of device clustering, which is quite fundamental to D2D network architecture [5], [6], [8]. To address these shortcomings, this paper develops a more accurate spatial model for cache-enabled clustered D2D networks. We focus on the cluster-centric content placement in out-band D2D communications, in which cellular and D2D transmissions do not interfere. More details are given next.

Contributions and outcomes. We develop a realistic and tractable spatial model for D2D networks in which the locations of devices are modeled by a Poisson cluster process. We study the problem of cluster-centric content placement in which we show that the collective performance of the network is improved if the content is cached towards the center of each cluster. In particular, we prove that if one has to choose one device per cluster to place all the content of interest for that cluster, it will be the device that is closest to the cluster center. Since placing all the content at a single device limits frequency reuse to one and may not also be feasible due to storage/energy constraints, we also consider a more general class of clustercentric content placement startegies in which the content of interest for the typical device is placed at the $k^{\text {th }}$ closest device to the cluster center. We then derive easy to use expressions for coverage probability and ASE as a function of $k$. As a baseline case, we derive the coverage and ASE for the network when the content of interest is placed at a randomly chosen device in each cluster. Our analysis concretely demonstrates significant 


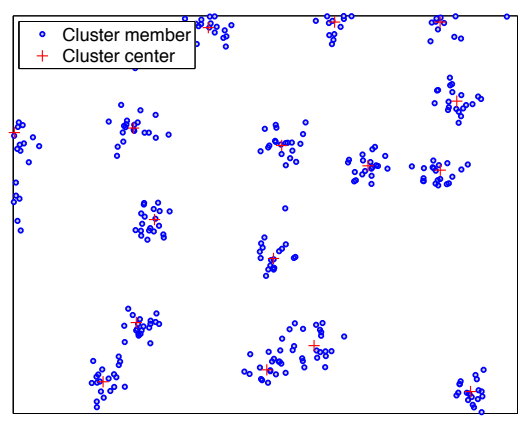

Fig. 1. Illustration of the proposed D2D network.

improvement in network performance when the content of interest for the typical device is biased to lie closer to the cluster center compared to the baseline random placement.

\section{SySTEM MOdEL}

We model the locations of the devices by a Poisson cluster process. As shown in Fig. 1, this point process is formed by the union of the cluster members (devices) which are scattered around their respective cluster centers. In our setup, similar to the Thomas cluster process, cluster centers $\{x\}$ are drawn from a homogeneous PPP $\Phi_{\mathrm{c}}$ and the cluster members are independent and identically distributed (i.i.d.) around each cluster center $x \in \Phi_{\mathrm{c}}$ according to a symmetric normal distribution with variance $\sigma^{2}$ [14]. Therefore, the density function of the cluster member location relative to its cluster center location, $a \in R^{2}$, is

$$
f_{A}(a)=\frac{1}{2 \pi \sigma^{2}} \exp \left(-\frac{\|a\|^{2}}{2 \sigma^{2}}\right) .
$$

Denoting the set of cluster members of the cluster at $x \in \Phi_{\mathrm{c}}$ by $\mathcal{N}^{x}$, we assume that the number of devices in each cluster is $N$, i.e., $\left|\mathcal{N}^{x}\right|=N, \forall x \in \Phi_{\mathrm{c}}$, where half of the devices are potential D2D-Txs. The set of simultaneously active devices in a cluster at $x \in \Phi_{\mathrm{c}}$ is denoted by $\mathcal{A}^{x} \subset \mathcal{N}^{x}$. The devices that form $\mathcal{A}^{x}$ are chosen uniformly at random from $\mathcal{N}^{x}$. The number of simultaneously active links (or D2D transmitters) $\left|\mathcal{A}^{x}\right|$ is modeled as a Poisson random variable with mean $\bar{m}$ conditioned on the fact that $\left|\mathcal{A}^{x}\right|$ is smaller than the total number of potential D2D-Txs inside the cluster $N^{\mathrm{t}}=N / 2$. Therefore, the complete set of transmitters in the whole network is denoted by $\Psi=\cup_{x \in \Phi_{c}} \mathcal{A}^{x}$. In terms of device access, we assume that the D2D links are formed between devices lying in the same cluster while the formation of direct links across clusters is not needed. This assumption can be justified by the fact that: (i) intra-cluster distances are typically much smaller than the inter-cluster distances, which makes it easier to establish direct links between the devices lying in the same cluster, and (ii) the devices in one cluster may not have information of interest to the devices of the other clusters. This assumption can be relaxed in some applications.

Now without loss of generality, we perform analysis on the randomly chosen D2D-Rx of interest located at the origin $o \in \mathbb{R}^{2}$ (termed typical device) inside the randomly chosen representative cluster $x_{0} \in \Phi_{\mathrm{c}}$. We assume D2D-Txs transmit with constant power $P_{\mathrm{d}}$ and the content of interest for the typical device is available at the device located at $a_{0}+x_{0}$. Hence, the received power at the typical device is

$$
P=P_{\mathrm{d}} h_{a_{0}}\left\|x_{0}+a_{0}\right\|^{-\alpha},
$$

where $h_{a_{0}} \sim \exp (1)$ models Rayleigh fading between D2DTx and D2D-Rx of interest and $\alpha>2$ is power-law path loss exponent. Now, the total interference experienced by D2D$\mathrm{Rx}$ of interest can be written as the sum of two independent terms. First, interference from the set of devices inside the representative cluster, say intra-cluster interference, at D2D$\mathrm{Rx}$ of interest can be expressed as

$$
I_{\text {intra }}=\sum_{a \in \mathcal{A}^{x_{0}} \backslash a_{0}} P_{\mathrm{d}} h_{a_{x_{0}}}\left\|x_{0}+a\right\|^{-\alpha} .
$$

Second, interference from devices outside the representative cluster, say inter-cluster interference is

$$
I_{\text {inter }}=\sum_{x \in \Phi_{\mathrm{c}} \backslash x_{0}} \sum_{a \in \mathcal{A}^{x}} P_{\mathrm{d}} h_{a_{x}}\|x+a\|^{-\alpha} .
$$

Now, the SIR experienced by D2D-Rx of interest at distance $R=\left\|x_{0}+a_{0}\right\|$ (a realization of $R$ will be denoted by $r$ ) from D2D-Tx of interest is:

$$
\operatorname{SIR}(r)=\frac{P_{\mathrm{d}} h_{a_{0}} r^{-\alpha}}{I_{\text {inter }}+I_{\text {intra }}} .
$$

Since the SIR expression above is not the function of transmit power, we can assume $P_{\mathrm{d}}=1$ without loss of generality.

\section{ASYNCHRONOUS CONTENT REUSE AND D2D COMMUNICATION}

\section{A. Coverage-optimal content placement}

We first study the coverage-optimal content placement problem in the proposed clustered D2D model. Note that while it is preferable to place the content required by each device at its immediately neighboring device, such device-centric content placement is not quite realistic. Therefore, we focus on the cluster-centric coverage-optimal content placement, where the goal is to place the content in such a way that it improves the collective performance of the whole network. In other words, instead of finding the optimal content placement for each device, we focus on placing content at a single location that will improve the overall performance of the whole cluster. We cast this problem as the coverage probability maximization problem, where coverage probability of a typical device is

$$
\mathrm{P}_{\mathrm{c}}=\mathbb{E}\left[\mathbf{1}\left\{\operatorname{SIR}\left(\left\|x_{0}+s_{k}\right\|\right)>\beta\right\}\right],
$$

where $\beta$ is the target SIR threshold, typical device is located at the origin, $x_{0}$ is the location of the cluster center, and $s_{k}$ is the location of the serving device relative to the cluster center. We order the devices in the representative cluster in terms of increasing distances from the cluster centers, i.e., $k=1$ and $k=N^{\mathrm{t}}$ correspond to the closest and farthest devices to the cluster center, respectively. The goal is to find the value of $k$ that maximizes coverage probability. The result is given in the next Lemma and the proof is given in Appendix A. 
Lemma 1. The optimal cache placement to maximize overall coverage probability in a given cluster is:

$$
\arg \max _{k \in\left\{1,2, \ldots, N^{\mathrm{t}}\right\}} \mathbb{E}\left[\mathbf{1}\left\{\operatorname{SIR}\left(\left\|x_{0}+s_{k}\right\|\right)>\beta\right\}\right]=1 \text {. }
$$

An intuitive interpretation of this result is that all the devices in a given cluster should be served by a device that is on an average closest to all of them. As proved formally in the above Lemma, this device is the one that is closest to the cluster center. However, the largest drawback of this policy is that it limits the frequency reuse capability of D2D networks by concentrating all the content at a single device. Besides, such a policy may be infeasible due to storage and energy constraints of devices. As a result, we define and explore the following two general classes of content placement policies.

- Case \#1: In this case, we assume that the content of interest for the typical device is available at the $k^{t h}$ closest device to the cluster center. This is useful because by tuning the value of $k$, we can bias the presence of content towards the cluster center or away from it.

- Case \#2: In this case, we assume that the content of interest for the typical device is placed at a device chosen uniformly at random from the same cluster. This will act as a baseline case for the performance comparisons.

We now derive coverage probability and ASE for the two cases.

\section{B. Coverage probability and ASE analysis}

Before going into the detailed analysis of coverage probability, it is important to understand how the selection of serving device impacts the intra-cluster interference. In Case \#1, serving device is the $k^{t h}$ closest device to the cluster center out of the total of $N^{\mathrm{t}}$ devices in the representative cluster. This means that the interferers can lie at any of the possible $N^{\mathrm{t}}$ locations except where the serving device is located (i.e., except the $k^{t h}$ closest location to the cluster center). The exact analysis of Laplace transform of interference corresponding to the Case \#1 is provided in the extended version of this paper [15]. Here, due to space limitations we ignore this dependence of the distances of the interfering links on $k$. In other words, we assume interferers to be normally distributed around the cluster centers independent of the serving device location. In Case \#2, however, the selection of serving device is done randomly, which does not impact the distribution of the interferers, thus leading to the exact analysis for this case. Even for Case \#1, we will notice in the numerical results section that the approximation is fairly tight. We now present the Laplace transform of intra-cluster interference, which is exact for Case \#2 and approximation for Case \#1.

Lemma 2. Laplace transform of the intra-cluster interference at the typical device, conditioned on the distance from the typical device to the cluster center, is

$$
\mathcal{L}_{I_{\text {intra }}}\left(s \mid \nu_{0}\right)=\sum_{k=0}^{N^{\mathrm{t}}-1}\left(M\left(w \mid \nu_{0}\right)\right)^{k} \frac{(\bar{m}-1)^{k} e^{-(\bar{m}-1)}}{k ! \xi}
$$

with $M\left(w \mid \nu_{0}\right)=\int_{0}^{\infty} \frac{1}{1+s w^{-\alpha}} f_{W}\left(w \mid \nu_{0}\right) \mathrm{d} w$. Assuming $\bar{m} \ll$ $N^{\mathrm{t}}$, we have

$$
\mathcal{L}_{I_{\text {intra }}}\left(s \mid \nu_{0}\right) \simeq \frac{1}{\xi} \exp \left(-(\bar{m}-1)\left(1-M\left(w \mid \nu_{0}\right)\right)\right),
$$

where $\xi=\sum_{j=0}^{N^{t}-1} \frac{(\bar{m}-1)^{j} e^{-(\bar{m}-1)}}{j !}$ and $f_{W}\left(w \mid \nu_{0}\right)=$ $\frac{w}{\sigma^{2}} \exp \left(-\frac{w^{2}+\nu_{0}^{2}}{2 \sigma^{2}}\right) I_{0}\left(\frac{w \nu_{0}}{\sigma^{2}}\right), \quad w>0$.

Proof. See Appendix D.

Note that in practice the average number of simultaneously active links is much smaller than the total number of potential transmitters $N^{\mathrm{t}}$ inside the cluster. Hence, the assumption of $\bar{m} \ll N^{\mathrm{t}}$ in Lemma 2 is reasonable which leads to a simpler expression (8) that is also very accurate. The Laplace transform expressions can be simplified further under the following assumption without loosing much accuracy.

Assumption 1 (Uncorrelated intra-cluster distances assumption). We assume that correlation due to the common distance $\nu_{o}=\left\|x_{0}\right\|$ between transmitting devices (intra-cluster interferers and D2D-Tx of interest) to the typical device is weak. Therefore, instead of considering the conditional intra-cluster distances (Rician distributed with density $f_{W}\left(w \mid \nu_{0}\right)$ ), we can consider their marginal distribution (Rayleigh):

$$
f_{W}(w)=\frac{w}{2 \sigma^{2}} \exp \left(-\frac{w^{2}}{4 \sigma^{2}}\right), \quad w>0 .
$$

Under this assumption, the approximation for Laplace transform of intra-cluster interference is given next.

Corollary 1. The Laplace transform of intra-cluster interference under Assumption 1 can be approximated as

$$
\tilde{\mathcal{L}}_{I_{\text {intra }}}(s) \simeq \frac{1}{\xi} \exp \left((1-\bar{m}) \int_{0}^{\infty} \frac{s w^{-\alpha}}{1+s w^{-\alpha}} f_{W}(w) \mathrm{d} w\right),
$$

where $\xi=\sum_{j=0}^{N^{\mathrm{t}}-1} \frac{(\bar{m}-1)^{j} e^{-(\bar{m}-1)}}{j !}$ and $f_{W}(w)$ given by (9).

We will use this approximation to provide easy to compute expression for coverage probability later in this section. We now derive the Laplace transform of inter-cluster interference.

Lemma 3. The Laplace transform of inter-cluster interference at D2D-Rx of interest in (3) is given by:

$$
\begin{array}{r}
\mathcal{L}_{I_{\text {inter }}}(s) \simeq \exp \left(-2 \pi \lambda_{\mathrm{c}} \int_{0}^{\infty}\left(1-\frac{1}{\eta} \exp \left(-\bar{m} \int_{0}^{\infty} \frac{s u^{-\alpha}}{1+s u^{-\alpha}}\right.\right.\right. \\
\left.\left.\left.f_{U}(u \mid \nu) \mathrm{d} u\right) \nu \mathrm{d} \nu\right)\right), \quad(11)
\end{array}
$$

where $\eta=\sum_{j=0}^{N^{\mathrm{t}}} \frac{\bar{m}^{j} e^{-\bar{m}}}{j !}$ and $f_{U}(u \mid \nu)=$ $\frac{u}{\sigma^{2}} \exp \left(-\frac{u^{2}+\nu^{2}}{2 \sigma^{2}}\right) I_{0}\left(\frac{u \nu}{\sigma^{2}}\right) \quad u>0$.

Proof. See Appendix E.

1) Coverage probability analysis of Case \#1: We first derive the distribution of serving distance for this case.

Lemma 4. Assuming the content of interest is available at the $k^{\text {th }}$ closest device to the cluster center, the density function of the serving distance $r=\left\|x_{0}+s_{k}\right\|$ conditioned on the distances $\nu_{0}=\left\|x_{0}\right\|$ and $t_{k}=\left\|s_{k}\right\|$ is

$$
\begin{aligned}
f_{R}\left(r \mid \nu_{0}, t_{k}\right) & =\frac{1}{\pi} \frac{r / \nu_{0} t_{k}}{\sqrt{1-\left(\frac{\nu_{0}^{2}+t_{k}^{2}-r^{2}}{2 \nu_{0} t_{k}}\right)^{2}}}, \quad r>0, \quad \text { with } \\
f_{V_{0}}\left(\nu_{0}\right) & =\frac{\nu_{0}}{\sigma^{2}} \exp \left(-\frac{\nu_{0}^{2}}{2 \sigma^{2}}\right), \quad \nu_{0}>0
\end{aligned}
$$




$$
f_{T_{k}}\left(t_{k}\right)=\frac{N^{\mathrm{t}} !}{(k-1) !\left(N^{\mathrm{t}}-k\right) !} F\left(t_{k}\right)^{k-1}\left(1-F\left(t_{k}\right)\right)^{N^{\mathrm{t}}-k} f\left(t_{k}\right)
$$

where $f\left(t_{k}\right)=\frac{t_{k}}{\sigma^{2}} \exp \left(-\frac{t_{k}^{2}}{2 \sigma^{2}}\right)$, and $F\left(t_{k}\right)=1-\exp \left(-\frac{t_{k}^{2}}{2 \sigma^{2}}\right)$. Proof. See Appendix B.

We now derive the coverage probability for Case \#1 in the following theorem.

Theorem 1. Assuming the file of interest is available at $k^{\text {th }}$ closet device to the cluster center, the coverage probability of the randomly chosen device inside the cluster is

$$
\begin{array}{r}
\mathrm{P}_{\mathrm{c}} \approx \int_{0}^{\infty} \int_{0}^{\infty} \int_{0}^{\infty} \mathcal{L}_{I_{\text {inter }}}\left(\beta r^{\alpha}\right) \mathcal{L}_{I_{\text {intra }}}\left(\beta r^{\alpha} \mid \nu_{0}\right) f_{R}\left(r \mid \nu_{0}, t_{k}\right) \\
\times f_{V_{0}}\left(\nu_{0}\right) f_{T_{k}}\left(t_{k}\right) \mathrm{d} r \mathrm{~d} \nu_{0} \mathrm{~d} t_{k}, \quad(15)
\end{array}
$$

where $f_{R}\left(r \mid \nu_{0}, t_{k}\right), f_{V_{0}}\left(\nu_{0}\right)$, and $f_{T_{k}}\left(t_{k}\right)$ are given by (12), (13), and (14) respectively.

Proof. From the definition of coverage probability, we have

$$
\begin{aligned}
& \mathrm{P}_{\mathrm{c}} \stackrel{(a)}{\approx} \mathbb{E}\left[\mathbb{P}\left\{\frac{h_{0 x_{0}} r^{-\alpha}}{I_{\text {inter }}+I_{\text {intra }}}>\beta \mid R\right\}\right] \\
& =\mathbb{E}_{T_{k}} \mathbb{E}_{V_{0}} \mathbb{E}_{R}\left[\mathbb{P}\left\{h_{0 x_{0}}>\beta r^{\alpha}\left(I_{\text {inter }}+I_{\text {intra }}\right) \mid R, V_{0}, T_{k}\right\}\right] \\
& \stackrel{(b)}{=} \mathbb{E}_{T_{k}} \mathbb{E}_{V_{0}} \mathbb{E}_{R}\left[\mathbb{E}\left[\exp \left(-\beta r^{\alpha}\left(I_{\text {inter }}+I_{\text {intra }}\right)\right) \mid R, V_{0}, T_{k}\right]\right]
\end{aligned}
$$

where approximation in $(a)$ is due to the fact that Laplace transform of intra-cluster interference given by Lemma 2 is an approximation for this case, and $(b)$ follows from Rayleigh fading assumption. The result follows from de-conditioning over serving distance $R$ given $\nu_{0}$ and $t_{k}$ using the serving link distribution given by (12), followed by de-conditioning over $\nu_{0}$ and $t_{k}$ given by (14) and (13) respectively.

Note that the coverage probability expression is an approximation because of $\mathcal{L}_{I_{\text {intra }}}\left(\beta r^{\alpha} \mid \nu_{0}\right)$ given by Lemma 2 , which is not exact for Case \#1, although it is exact for Case \#2. Nevertheless, this approximation gets tight as the value of $k$ increases. Also note that the exact results are available in the extended version of this paper [15]. Further, while the coverage probability expression seems a bit involved, it can be easily solved by Quasi-Monte Carlo numerical integration methods (integrals are essentially expectations in this case) [16].

We now derive an easy-to-compute expression for coverage probability under Assumption 1. Using the approximation corresponding to the Laplace transform of the intra-cluster interference in Corollary 1, the coverage probability for Case \#1 can be approximated as follows.

Corollary 2. Under Assumption 1, coverage probability for Case \#1 can be approximated as $\mathrm{P}_{\mathrm{c}} \simeq$

$$
\int_{0}^{\infty} \int_{0}^{\infty} \mathcal{L}_{I_{\text {inter }}}\left(\beta r^{\alpha}\right) \tilde{\mathcal{L}}_{I_{\text {intra }}}\left(\beta r^{\alpha}\right) f_{R}\left(r \mid t_{k}\right) f_{T_{k}}\left(t_{k}\right) \mathrm{d} r \mathrm{~d} t_{k}
$$

where $f_{R}\left(r \mid t_{k}\right)=\frac{r}{\sigma^{2}} \exp \left(-\frac{r^{2}+t_{k}^{2}}{2 \sigma^{2}}\right) I_{0}\left(\frac{r t_{k}}{\sigma^{2}}\right)$, and $f_{T_{k}}\left(t_{k}\right)$ is given by (14).

Proof. See Appendix C.

We will validate the tightness of the approximation in the numerical result section.
2) Coverage probability analysis of Case \#2: We now derive the coverage probability of a typical device when its content of interest is available inside the cluster at random.

Theorem 2. Assuming content is available inside the cluster at random, the coverage probability at the typical device is

$\mathrm{P}_{\mathrm{c}}=\int_{0}^{\infty} \int_{0}^{\infty} \mathcal{L}_{I_{\text {inter }}}\left(\beta r^{\alpha}\right) \mathcal{L}_{I_{\text {intra }}}\left(\beta r^{\alpha} \mid \nu_{0}\right) f_{R}\left(r \mid \nu_{0}\right) f_{V_{0}}\left(\nu_{0}\right) \mathrm{d} r \mathrm{~d} \nu_{0}$,

where $f_{R}\left(r \mid \nu_{0}\right)=\frac{r}{\sigma^{2}} \exp \left(-\frac{r^{2}+\nu_{0}^{2}}{2 \sigma^{2}}\right) I_{0}\left(\frac{r \nu_{0}}{\sigma^{2}}\right)$, and $f_{V_{0}}\left(\nu_{0}\right)=$ $\frac{\nu_{0}}{\sigma^{2}} \exp \left(-\frac{\nu_{0}^{2}}{2 \sigma^{2}}\right)$.

Proof. The proof follows on the same line as Theorem 1. The density function of serving distance $r=\left\|x_{0}+a_{0}\right\|$ conditioned on $\nu_{0}=\left\|x_{0}\right\|$ can be derived on the same lines as Corollary 2 . Coverage probability of Case \#2 is computed at a randomly chosen device whose location relative to the cluster center is sampled from a Gaussian distribution in $\mathbb{R}^{2}$. Hence $\nu_{0}=\left\|x_{0}\right\|$ simply follows Rayleigh distribution.

3) ASE analysis: Assuming all D2D-Txs $(z \in \Psi)$ use Gaussian codebooks for their transmissions, the average number of bits transmitted per unit time per unit bandwidth per unit area, termed area spectral efficiency (ASE), is

$$
\mathrm{ASE}=\lambda \log _{2}(1+\beta) \mathbb{E}_{\{z\},\left\{h_{z}\right\}}[\mathbf{1}\{\operatorname{SIR}(r)>\beta\},
$$

where $\lambda$ is the number of simultaneously active transmitters per unit area. This definition can be easily specialized to our setup in the following theorem.

Theorem 3. The ASE corresponding to the two cases of interest is given by:

$$
\mathrm{ASE}=\bar{m} \lambda_{\mathrm{c}} \log _{2}(1+\beta) \mathrm{P}_{\mathrm{c}},
$$

where $\mathrm{P}_{c}$ is given by (15) for Case \#1 and (17) for Case $\# 2$. Here, $\bar{m} \lambda_{\mathrm{c}}$ denotes the average number of simultaneously active D2D-Txs.

Note that computing ASE for specific $k$ in Case \#1 is slightly simplistic because it assumes that for any receiver, the transmitting device is the $k^{t h}$ closest device to the cluster center, which is strictly true only for the typical device. Nevertheless, understanding the trends as a function of $k$ is still useful. More discussion will be provided in the next section.

\section{RESULTS AND DISCUSSION}

Validation of results: In this section, we validate the accuracy of the analytical results, and tightness of the approximations by means of simulation. In all simulation, the locations of cluster centers are a realization of a PPP and the devices are normally scattered around them. As shown in Fig. 2, the analytical result for Case \#1 given by Theorem 1, when file of interest is available at the closest device to the cluster center $(k=1)$, provides a very tight approximation for the simulation result. The result also shows that the approximation for coverage probability in Case \#1 derived under Assumption 1 given by Corollary 2 is fairly tight. This shows that the easy to compute approximate expression can very well be used as the proxy for coverage probability. For Case \#2, 


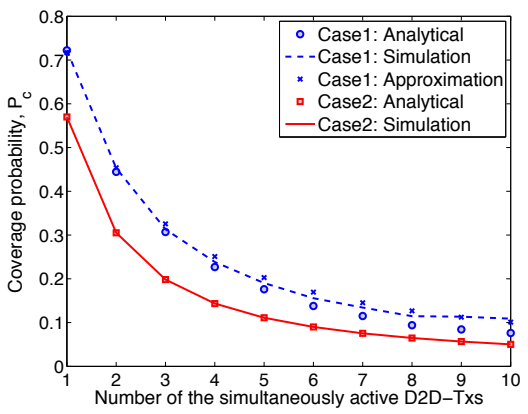

Fig. 2. Coverage probability versus number of simultaneously active D2DTxs when $\sigma=30, \lambda_{\mathrm{c}}=50$ clusters $/ \mathrm{km}^{2}, N^{\mathrm{t}}=40$, and $\beta=0 \mathrm{~dB}$.

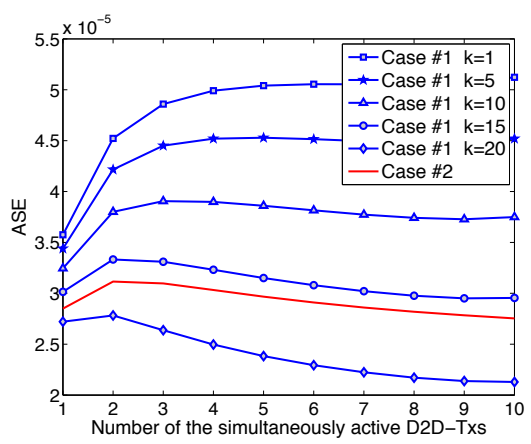

Fig. 3. ASE versus number of simultaneously active D2D-Txs when $\sigma=30$, $\lambda_{\mathrm{c}}=50$ clusters $/ \mathrm{km}^{2}, N^{\mathrm{t}}=30$, and $\beta=0 \mathrm{~dB}$.

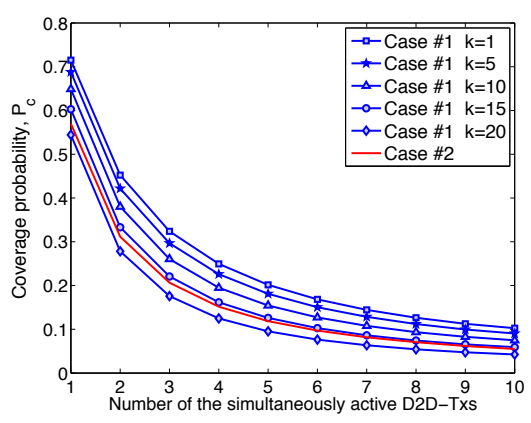

Fig. 4. Coverage probability versus number of simultaneously active D2DTxs when $\sigma=30, \lambda_{\mathrm{c}}=50$ clusters $/ \mathrm{km}^{2}, N^{\mathrm{t}}=30$, and $\beta=0 \mathrm{~dB}$.

the simulation and analytical results perfectly match, thus confirming the accuracy of analysis.

Coverage-optimal content placement: Note that there is a clear trade off between the optimal number of simultaneously active D2D-Txs and the resulting interference power. While increasing the number of simultaneously active transmitters potentially increases ASE, it also increases interference. As shown in Fig. 3, the optimal number of simultaneously active D2D-Txs increases with the decrease in distance from the D2D-Tx of interest to the cluster center (i.e., decreasing $k$ ). Fig. 3 and Fig. 4 show that coverage probability and ASE are optimum when the content of interest for the typical device is available at the closest device to the cluster center. Also, the results show that optimal content placement leads to a significant improvement in both coverage probability and ASE compared to random content placement (Case \#2). On the contrary, it can be seen that the both coverage probability and ASE may become worse than the random placement case when the content is cached far from the cluster center.

\section{CONClusion}

In this paper, we have developed a realistic framework for the modeling and analysis of cache-enabled D2D networks. Modeling the D2D network as a Poisson cluster process, we have concretely shown that for the collective network performance improvement in terms of coverage probability, it is optimal to cache the content at the device closest to the cluster center. However, since this policy may limit the frequency reuse inside the cluster to one, we have also explored a more general caching strategy in which the content of interest for the typical device is placed at the $k^{t h}$ closest device to the cluster center. Using tools from stochastic geometry, we derived the coverage and ASE for these strategies and compared them to the case when the content is placed at random in the cluster.

\section{APPENDIX}

\section{A. Proof of Lemma 1}

Denote the set of potential D2D-TXs inside a representative cluster by $\mathcal{N}_{\mathrm{t}}^{x_{0}} \in\left\{1,2, \ldots, N^{\mathrm{t}}\right\}$. Assuming the file of interest is located at $x_{0}+s_{k}$, the coverage-optimal cache placement is

$$
\begin{aligned}
& k^{*}=\arg \max _{k \in \mathcal{N}_{\mathrm{t}}^{x_{0}}} \mathbb{E}\left[\mathbf{1}\left\{\operatorname{SIR}\left(\left\|x_{0}+s_{k}\right\|\right)>\beta\right\}\right] \\
& \stackrel{(a)}{=} \arg \max _{k \in \mathcal{N}_{\mathrm{t}}^{x_{0}}} \mathbb{E}\left[\frac{h_{a_{0}}\left\|x_{0}+s_{k}\right\|^{-\alpha}}{I-h_{a_{0}}\left\|x_{0}+s_{k}\right\|^{-\alpha}}>\beta\right] \\
& \quad=\arg \max _{k \in \mathcal{N}_{\mathrm{t}}^{x_{0}}} \mathbb{E}\left[\frac{\left\|x_{0}+s_{k}\right\|^{-\alpha}}{\frac{1}{h_{a_{0}}} I-\left\|x_{0}+s_{k}\right\|^{-\alpha}}>\beta\right] \\
& \stackrel{(b)}{=} \arg \max _{k \in \mathcal{N}_{\mathrm{t}}^{x_{0}}} \mathbb{E}\left[X_{k}>\beta\right]
\end{aligned}
$$

where $I$ in $(a)$ is the total received power at the typical device from all the transmitters in the network, and $X_{k}$ is defined as the received SIR from the $k^{t h}$ closest serving device for the ease of notation. Since $I$ is not the function of $k, X_{k} \geq_{\text {st }} X_{j}$ whenever $\left\|x_{0}+s_{j}\right\| \geq_{\text {st }}\left\|x_{0}+s_{k}\right\|$, where $\geq_{\text {st }}$ denotes first order stochastic dominance (or usual stochastic order). Note that since $x_{0}$ is sampled from zero mean complex Gaussian random variable in $\mathbb{R}^{2}$, the density function of $r=\left\|x_{0}+s_{k}\right\|$ conditioned on $t_{k}=\left\|s_{k}\right\|$ follows Rician distribution with $\mathrm{CDF} F_{R}\left(r \mid t_{k}\right)=1-Q_{1}\left(\frac{t_{k}}{\sigma}, \frac{r}{\sigma}\right)$. It turns out that $Q_{1}(\alpha, \beta)$ is monotonically increasing in $\alpha$ [17, Property 11], which implies $Q_{1}\left(\frac{t_{k}}{\sigma}, \frac{r}{\sigma}\right)$ is monotonically increasing in $t_{k}$, which implies $R\left(t_{k}\right) \leq_{\text {st }} R\left(t_{k}+1\right)$. This implies $\left\|x_{0}+s_{j}\right\| \geq_{\text {st }}\left\|x_{0}+s_{1}\right\|$ $\forall j \neq 1$, which completes the proof.

\section{B. Proof of Lemma 4}

The CDF of serving distance $r=\left\|x_{0}+s_{k}\right\|$ conditioned on $v_{0}=\left\|x_{0}\right\|$ and $t_{k}=\left\|s_{k}\right\|$ can be derived as follows:

$$
\begin{aligned}
F_{R}\left(r \mid \nu_{0}, t_{k}\right) & =\mathbb{P}\left[R<r \mid \nu_{0}, t_{k}\right] \\
& \stackrel{(a)}{=} \mathbb{P}\left[\nu_{0}^{2}+t_{k}^{2}-2 \nu_{0} t_{k} \cos (\theta) \leq r^{2}\right] \\
& =\mathbb{P}\left[\cos (\theta) \geq \frac{\nu_{0}^{2}+t_{k}^{2}-r^{2}}{2 \nu_{0} t_{k}} \mid \nu_{0}, t_{k}\right]
\end{aligned}
$$




$$
\begin{aligned}
& \stackrel{(b)}{=} \mathbb{P}\left[\theta<\cos ^{-1}\left[\frac{\nu_{0}^{2}+t_{k}^{2}-r^{2}}{2 \nu_{0} t_{k}}\right] \mid \nu_{0}, t_{k}\right] \\
& \stackrel{(c)}{=} \frac{1}{\pi} \cos ^{-1}\left[\frac{\nu_{0}^{2}+t_{k}^{2}-r^{2}}{2 \nu_{0} t_{k}} \mid \nu_{0}, t_{k}\right],
\end{aligned}
$$

where (a) follows from the cosine law, (b) follows from the fact that $\cos ^{-1}$ is monotonically decreasing function, and (c) follows from the fact that $\theta \sim \operatorname{Unif}[0, \pi]$. The result follows by taking the derivative of the CDF.

\section{Proof of Corollary 2}

Under Assumption 1, the correlation corresponding to the common distance $\nu_{0}=\left\|x_{0}\right\|$ is ignored and hence the Laplace transform of the interference can be approximated by Corollary 1. Furthermore, the density function of serving distance $r=\left\|x_{0}+s_{k}\right\|$ needs to be evaluated only conditioned on the distance of D2D-Tx of interest (i.e., $k^{\text {th }}$ closest device) to the cluster center $t_{k}=\left\|s_{k}\right\|$. Hence, the density function of $f_{R}\left(r \mid t_{k}\right)$ follows Rician distribution owing to the fact that $x_{0}$ is zero mean complex Gaussian random variable.

\section{Proof of Lemma 2}

The Laplace transform of intra-cluster interference can be derived as: $\mathcal{L}_{I_{\text {intra }}}\left(s \mid \nu_{0}\right)=\mathbb{E}\left[\exp \left(-s I_{\text {intra }}\right)\right]$

$$
\begin{aligned}
& \stackrel{(a)}{=} \mathbb{E}\left[\exp \left(-s \sum_{a \in \mathcal{A}^{x_{0}} \backslash a_{0}} h_{a_{x_{0}}}\left\|x_{0}+a\right\|^{-\alpha}\right]\right. \\
& =\mathbb{E}_{\mathcal{A}^{x_{0}}}\left[\prod_{a \in \mathcal{A}^{x_{0}} \backslash a_{0}} \mathbb{E}_{h_{a_{x_{0}}}}\left[\exp \left(-s h_{a_{x_{0}}}\left\|x_{0}+a\right\|^{-\alpha}\right)\right]\right] \\
& \stackrel{(b)}{=} \mathbb{E}_{\mathcal{A}^{x_{0}}}\left[\prod_{a \in \mathcal{A}^{x_{0}} \backslash a_{0}} \frac{1}{1+s\left\|a+x_{0}\right\|^{-\alpha}}\right] \\
& \stackrel{(c)}{=} \sum_{k=0}^{N^{\mathrm{t}}-1}\left(\int_{\mathbb{R}^{2}} \frac{1}{1+s\left\|a+x_{0}\right\|^{-\alpha}} f_{A}(a) \mathrm{d} a\right)^{k} \mathbb{P}\left(K=k \mid K<N^{\mathrm{t}}\right)
\end{aligned}
$$

where (a) follows from the definition of Laplace transform of interference, (b) follows from the fact that $h_{a_{x_{0}}}$ is exponential distributed with mean 1 , (c) follows from the fact that locations of devices conditioned on the location of cluster center are independent, and the result follows from converting Cartesian to polar coordinates with some algebraic manipulations. Now under the assumption $\bar{m} \ll N^{\mathrm{t}}$, the Laplace transform of intracluster can be approximated as:

$$
\simeq \frac{1}{\xi} \exp \left(-(\bar{m}-1) \int_{0}^{\infty} \frac{s w^{-\alpha}}{1+s w^{-\alpha}} f_{W}\left(w \mid \nu_{0}\right)\right) .
$$

The density function of $f_{W}\left(w \mid \nu_{0}\right)$ is formally derived in the extended version of this paper [18].

\section{E. Proof of Lemma 3}

The Laplace transform is $\mathcal{L}_{I_{\text {inter }}}(s)=$

$$
\begin{aligned}
& =\mathbb{E}\left[\exp \left(-s \sum_{x \in \Phi_{\mathrm{c}} \backslash x_{0}} \sum_{a \in \mathcal{A}^{x}} h_{a_{x}}\|x+a\|^{-\alpha}\right)\right] \\
& \stackrel{(a)}{=} \mathbb{E}_{\Phi_{\mathrm{c}}}\left[\prod_{x \in \Phi_{\mathrm{c}} \backslash x_{0}} \mathbb{E}_{\mathcal{A}^{x}}\left[\prod_{a \in \mathcal{A}^{x}} \frac{1}{1+s\|x+a\|^{-\alpha}} \mid x\right]\right. \\
& \stackrel{(b)}{\simeq} \mathbb{E}_{\Phi_{\mathrm{c}}}\left[\prod_{x \in \Phi_{\mathrm{c}} \backslash x_{0}} \frac{1}{\eta} \exp \left(-\bar{m} \int_{0}^{\infty} \frac{s u^{-\alpha}}{1+s u^{-\alpha}} f_{U}(u \mid \nu) \mathrm{d} u\right)\right]
\end{aligned}
$$

$$
\begin{gathered}
\stackrel{(c)}{=} \exp \left(-2 \pi \lambda_{c} \int_{0}^{\infty}\left(1-\frac{1}{\eta} \exp \left(-\bar{m} \int_{0}^{\infty} \frac{s u^{-\alpha}}{1+s u^{-\alpha}}\right.\right.\right. \\
\left.\left.\left.f_{U}(u \mid \nu) \mathrm{d} u\right)\right) \nu \mathrm{d} \nu\right)
\end{gathered}
$$

where (a) follows from the fact that $h_{a_{x}} \sim \exp (1)$, (b) follows on the same lines as proof of Lemma 2 where $\eta=\sum_{j=0}^{N^{\mathrm{t}}} \frac{\bar{m}^{j} e^{-\bar{m}}}{j !}$, and (c) follows from probability generating functional of PPP [19].

\section{REFERENCES}

[1] L. Song, D. Niyato, Z. Han, and E. Hossain, Wireless Device-to-Device Communications and Networks. Cambridge University Press, 2015.

[2] M. Cha, H. Kwak, P. Rodriguez, Y.-Y. Ahn, and S. Moon, "I Tube, You Tube, Everybody Tubes: analyzing the world's largest user generated content video system," in Proc., ACM Intl. Conf. on Special Interest Group on Data Commun. (SIGCOMM), 2007.

[3] K. Shanmugam, N. Golrezaei, A. G. Dimakis, A. F. Molisch, and G. Caire, "Femtocaching: Wireless content delivery through distributed caching helpers," IEEE Trans. on Info. Theory, vol. 59, no. 12, pp. 8402-8413, Dec. 2013

[4] A. F. Molisch, G. Caire, D. Ott, J. R. Foerster, D. Bethanabhotla, and M. Ji, "Caching eliminates the wireless bottleneck in video aware wireless networks," Advances in Electrical Engineering, Nov. 2014.

[5] M. Ji, G. Caire, and A. F. Molisch, "Wireless device-to-device caching networks: Basic principles and system performance," submitted to IEEE Journal on Sel. Areas in Commun., 2014, available online: arxiv.org/abs/1305.5216.

[6] N. Golrezaei, P. Mansourifard, A. Molisch, and A. Dimakis, "Basestation assisted device-to-device communications for high-throughput wireless video networks," IEEE Trans. on Wireless Commun., vol. 13, no. 7, pp. 3665-3676, Jul. 2014.

[7] S. Krishnan and H. S. Dhillon, "Distributed caching in device-todevice networks: A stochastic geometry perspective," to appear in Proc. Asilomar, Pacific Grove, CA, Nov. 2015.

[8] Y. Zhang, E. Pan, L. Song, W. Saad, Z. Dawy, and Z. Han, "Social network aware device-to-device communication in wireless networks," IEEE Trans. on Wireless Commun., vol. 14, no. 1, pp. 177-190, Jan. 2015.

[9] N. Lee, X. Lin, J. G. Andrews, and R. W. Heath Jr., "Power control for d2d underlaid cellular networks: Modeling, algorithms, and analysis," IEEE Journal on Sel. Areas in Commun., vol. 33, no. 1, pp. 1-13, Jan. 2015.

[10] A. H. Sakr and E. Hossain, "Cognitive and energy harvesting-based D2D communication in cellular networks: Stochastic geometry modeling and analysis," IEEE Trans. on Commun., vol. 63, no. 5, pp. 1867-1880, May. 2015.

[11] H. Sun, M. Wildemeersch, M. Sheng, and T. Q. Quek, "D2D enhanced heterogeneous cellular networks with dynamic TDD," to appear, IEEE Trans. on Wireless Commun., 2015, available online: arxiv.org/abs/1406.2752.

[12] X. Lin, R. Ratasuk, A. Ghosh, and J. G. Andrews, "Modeling, analysis and optimization of multicast device-to-device transmissions," IEEE Trans. on Wireless Commun., vol. 13, no. 8, pp. 4346-4359, Aug. 2014.

[13] H. ElSawy and E. Hossain, "Analytical modeling of mode selection and power control for underlay D2D communication in cellular networks," IEEE Trans. on Commun., vol. 62, no. 11, pp. 4147-4161, Nov. 2014.

[14] R. K. Ganti and M. Haenggi, "Interference and outage in clustered wireless ad hoc networks," IEEE Trans. on Info. Theory, vol. 55, no. 9, pp. 4067-4086, Sep. 2009.

[15] M. Afshang, H. S. Dhillon, and P. H. J. Chong, "Fundamentals of cluster-centric content placement in cache-enabled device-to-device networks," submitted to IEEE Trans. on Commun., 2015, available online: arxiv.org/abs/1509.04747.

[16] R. E. Caflisch, "Monte carlo and quasi-monte carlo methods," Acta Numerica, vol. 7, pp. 1-49, Jan. 1998.

[17] R. T. Short, "Computation of rice and noncentral chi-squared probabilities," Apr. 2012.

[18] M. Afshang, H. S. Dhillon, and P. H. J. Chong, "Modeling and performance analysis of clustered device-to-device networks," submitted to IEEE Trans. on Wireless Commun., available online: arxiv.org/abs/1508.02668.

[19] D. Stoyan, W. S. Kendall, and J. Mecke, Stochastic Geometry and its Applications, 2nd ed. New York: John Wiley and Sons, 1995. 